\title{
Physique des fluides aux échelles microscopiques pour l'ingénierie des microsystèmes : l'apprentissage par la simulation
}

\author{
Simon Laurette ${ }^{a}$, Renaud Dufour ${ }^{a b}$, Farida Brendriaa ${ }^{a}$, Vincent Thomy ${ }^{* a}$ et Nour-Eddine \\ Bourzgui $^{a}$
}

\author{
${ }^{a}$ Institut d'Electronique, de Microélectronique et de Nanotechnologie (IEMN) - UMR CNRS 8520, Université Lille 1, \\ Villeneuve d'Ascq, FRANCE; E-mail : vincent.thomy@iemn.univ-lille1.fr \\ ${ }^{b}$ Institut Supérieur de l'Electronique et du Numérique (ISEN) - Lille, FRANCE \\ http ://master-mint.univ-lille1.fr/ \\ http ://biomems.iemn.univ-lille1.fr/ \\ http ://m2t-iemn.univ-lille1.fr/
}

La microfluidique est un domaine en pleine expansion. Ses multiples applications expliquent l'engouement actuel pour cette thématique de recherche. Elles peuvent concerner l'étude de phénomènes physiques, la chimie analytique ou encore la biologie moléculaire. Le développement de cette discipline nécessite notamment des centres de fabrication microélectronique de type "salle blanche". Nous l'avons donc introduite dans les enseignements du Master Micro et NanoTechnologies de l'Université de Lille 1 dès la première année sous forme de cours, TDs de simulations et TP en salle blanche. La série de TDs de simulations destinés à comprendre les enjeux fondamentaux de l'écoulement des fluides aux petites dimensions est ici présentée. Au cours des séances, les problématiques de l'écoulement laminaire, des effets capillaires et des phénomènes de diffusion sont abordées. Elles permetteront à l'étudiant d'acquérir le bagage théorique nécessaire pour la poursuite d'études dans le développement d'applications microfluidiques comme les "Laboratoires sur Puce".

\section{Introduction}

En 1642, Blaise Pascal inventa l'un des plus fameux ancêtre de l'ordinateur : la Pascaline. Quelques années plus tard, il s'intéressa à la mécanique des fluides et au concept de pression atmosphérique dans deux ouvrages : Expériences nouvelles touchant le vide [1] et Récit de la grande expérience de l'équilibre des liqueurs [2]. Se doutait-il que ces contributions convergeraient 3 siècles plus tard?

Grâce aux progrès de la miniaturisation de l'électronique lors de la deuxième moitié du XXème siècle, les puissances de calcul des ordinateurs se sont démultipliées alors que leur encombrement s'est réduit. La simulation, basée sur le traitement numérique des équations fondamentales, s'est alors imposée comme un moyen efficace pour la prévision des comporte- ments physiques de systèmes. Là où le traitement analytique rencontre des difficultés à formuler des solutions, la simulation numérique est capable de fournir des prévisions approchées de bonne qualité. Ainsi l'électromagnétisme, la mécanique, l'électronique ou encore la chimie ont recours aux logiciels de simulation multiphysique pour appréhender certaines situations expérimentales.

Mais la miniaturisation a permis d'autres avancées que la compacité et la performance des calculateurs. En effet, grâce aux techniques mises au point pour la fabrication de composants micro ou nanométriques dans des environnements contrôlés (salles blanches), de nombreux systèmes physiques ont pu être fabriqués : on parle de microsystèmes [3]. L'accéléromètre qui commande le déclenchement de l'airbag en est un, tout comme les têtes d'impression jet d'encre. 
Une perspective prometteuse de ces microsystèmes est leur application à l'analyse biologique [4]. Une part importante de la recherche est actuellement consacrée aux "Laboratoires sur Puce", dont l'objectif est l'intégration de l'analyse de fluides biologiques. Temps d'analyse et coûts réduits, auto-contrôle des patients à domicile, augmentation de la sensibilité, nombreuses sont les avancées que de tels laboratoires sont susceptibles d'apporter [5].

Pour concevoir de tels systèmes, appréhender la physique du transport des fluides aux échelles microscopiques est indispensable. En effet, si le transport du fluide à analyser (le sang par exemple) s'effectue dans des canaux de $100 \mu \mathrm{m}$ de largeur, les propriétés de l'écoulement sont largement différentes de celles à notre échelle [6]. De même, des effets quasiment ignorés à nos échelles deviennent prépondérant comme la capillarité ou la diffusion. Tout un pan de la recherche s'attelle donc à décrire la "microfluidique" ou le comportement des fluides aux "petites échelles" [7].

Malheureusement, les équations régissant les comportements des fluides (équation de Navier Stokes, de diffusion...) sont en général complexes et couplées. Leur résolution analytique est rarement triviale et donc, l'utilisation de logiciels de simulation dans ces domaines est souvent pratiquée.

Sur les pas des travaux pionniers de Pascal, la convergence entre la mécanique des fluide, les investigations technologiques et l'utilisation de la simulation se présente comme un nouveau champ de recherche et d'applications. En découle donc un enseignement à la fois multiphysique et multidisciplinaire.

La microfluidique étant en passe de devenir une branche de plus en plus représentée de la microélectronique, son enseignement à des étudiants de cursus de type EEA/SPI ou physique a été réfléchi au sein du Master Micro et NanoTechnologies à l'université de Lille 1. Une formule "cours, TD de simulations et TP expérimentaux" a été retenue. Nous proposons ici de décrire le déroulement des séances de TD dont l'objectif a été double : introduire un logiciel de simulation basé sur la méthode des éléments finis et faire resentir aux étudiants les différences entre écoulements aux échelles macroscopique et microfluidique. Trois séances (3h-4h4h) de TDs ont eu lieu. Pour travailler sur ces aspects, nous avons utilisé le logiciel de simulation multiphysique COMSOL (v4.0) :

- TD1 : prise en main de COMSOL et généralités sur les écoulements laminaires;

- TD2 : effets capillaires en microfluidique;

- TD3 : le problème du mélange en microfluidique ;

Le choix du logiciel a été guidé par le fait qu'il permet une prise en main rapide par les étudiants et qu'il ne nécessite pas de prérequis en matière de simulation numérique. De plus, de par le caractère multiphysique de COMSOL, les connaissances acquises sur l'utilisation du logiciel pourront être mises à profit par les étudiants pour d'autres problématiques scientifiques. Ce logiciel est en effet largement utilisé dans les laboratoires académiques, par des équipes de thématiques différentes. L'objet de la présente communication est la description du déroulement de ces TDs ainsi que les objectifs pédagogiques. Les énoncés ne sont pas fournis mais sont disponibles auprès des auteurs. Les modules nécessaires lors de ces séances sont "Laminar Flow" (TD1 et TD3), "Laminar Two-Phase Flow, Phase Field" (TD2) et "Transport of Diluted Species" (TD3).

\section{Ecoulements laminaires avec le logiciel COMSOL}

L'objectif du premier TD est double : utiliser un logiciel de simulation multiphysique et l'appliquer à l'écoulement laminaire dans un microcanal. De manière générale, toute simulation effectuée avec COMSOL se déroule suivant le synoptique suivant :

- Définition du type de problème (choix et chargement des équations à résoudre) ;

- Définition de la géométrie du problème;

- Chargement des matériaux utilisés;

- Définition des propriétés physiques du problème (conditions aux limites...);

- Définition du maillage (discrétisation des équations différentielles);

- Résolution du problème ;

- Traitement des résultats (post-traitement).

L'étude consiste en la résolution bidimensionnelle de l'équation de Navier Stokes dans le cas d'un microcanal de longueur $100 \mu \mathrm{m}$, et de section carrée de côté $10 \mu \mathrm{m}$ (module "Laminar Flow" de COMSOL). Une différence de pression est appliquée entre l'entrée et la sortie, et les murs du microcanal sont caractérisés par une vitesse nulle (non glissement). La première étape du TD est de déterminer le profil du champ des vitesses 


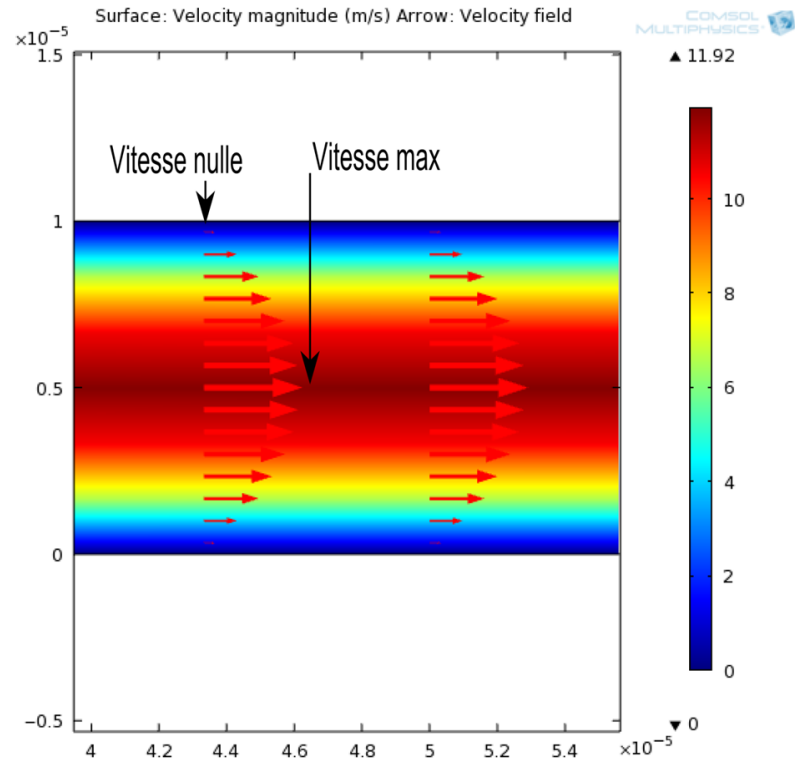

FIgURE 1 - Champ de vitesse parabolique dans le microcanal - Ecoulement de Poiseuille

dans le canal. Il est représenté en Figure 1. Toutes les figures de cette communication sont tirées des compte-rendus des étudiants.

La visualisation du profil parabolique de la vitesse est immédiate. Une discussion avec les étudiants est alors entamée sur le rôle prépondérant de la viscosité, responsable d'un tel écoulement qualifié de laminaire et largement rencontré en microfluidique. Le profil des vitesses peut également être tracé grâce à la mesure du champ des vitesses sur une coupe transverse. Il est représenté en Figure 2 et permet une discussion sur l'influence du choix du maillage, compromis entre précision des résultats et temps de calcul. Dans le cas de la présente étude stationnaire en 2 dimensions, un maillage très fin peut être choisi.

Norme de la vitesse $(\mathrm{m} / \mathrm{s})$

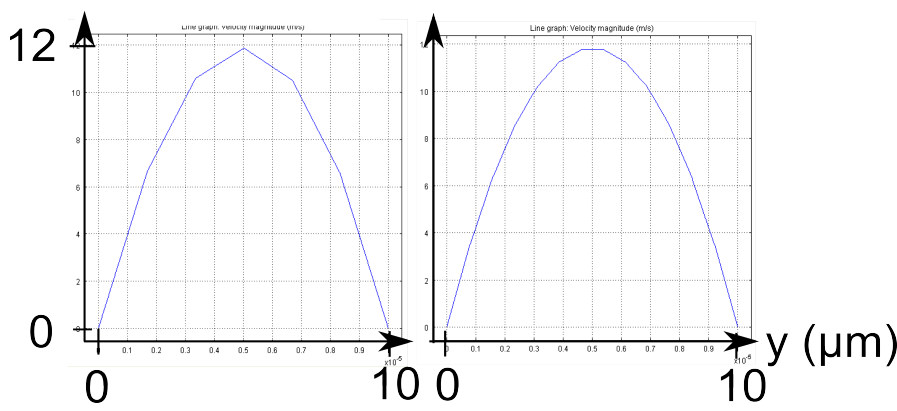

FIgURE 2 - Norme du champ de vitesses en fonction de la position verticale $y$ dans le microcanal. Influence du maillage (maillage plus fin pour la courbe de droite).
Une fois la notion de profil de vitesse parabolique abordée, l'étude se concentre sur la détermination du champ de pression au sein du microcanal. La simulation (Figure 3) montre une décroissance linéaire de la pression au sein du microcanal, dans le sens de la longueur. En réalité, près de l'entrée du canal l'évolution de la pression n'est pas totalement linéaire, ce qui permet d'aborder la notion "d'effets de bords" lors de la simulation. A ce stade, il est demandé aux étudiants d'établir une analogie entre le profil observé et l'évolution du potentiel électrique dans une résistance ou un potentiomètre. Le doigt est ainsi pointé sur l'analogie microfluidique/électrocinétique avec la dualité pression/tension. Le but de la suite du TD est d'étudier les limites de cette similitude.

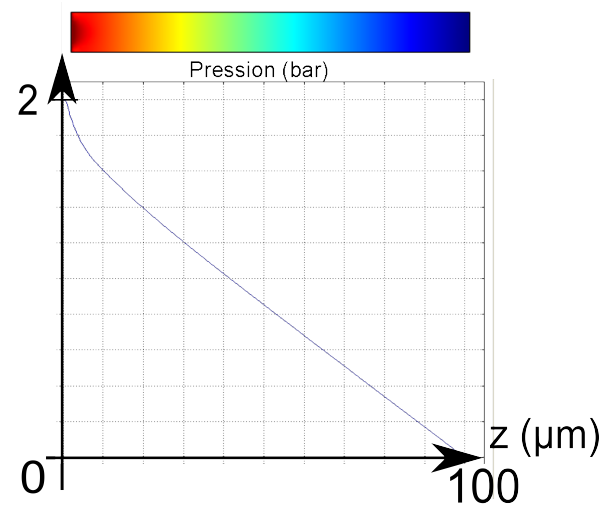

FIgURE 3 - Décroissance linéaire de la pression le long du microcanal

Pour cela, il est demandé aux étudiants de déterminer le débit volumique $D$ de l'écoulement en intégrant la champ de vitesse sur une section du microcanal. L'évolution du débit dans le microcanal est tracée en fonction du différentiel de pression $\Delta P$ entre l'entrée et la sortie. Le résultat est une évolution linéaire, qui est confrontée à la loi d'Ohm (Equation 1) de l'électronique. Le débit volumique correspond à l'intensité $I$ et on appelle résistance hydraulique $R_{h}$ le coefficient de proportionnalité entre le débit et la différence de pression.

$$
\begin{gathered}
\Delta V=R I \\
\Delta P=R_{h} D
\end{gathered}
$$

Le microcanal rempli d'eau se comporte donc comme une résistance pour l'écoulement laminaire du fluide. Lors de l'étape suivante du TD, les étudiants doivent déterminer quelles grandeurs influent sur la résistance hydraulique. Pour cela, on leur propose de faire varier la longueur du microcanal ainsi que la viscosité du 
fluide. En déterminant la résistance hydraulique dans chaque cas, ils s'aperçoivent que la résistance hydraulique est proportionnelle, par un facteur $k$, à la viscosité $\eta$ du fluide ainsi qu'à la longueur $L$ du microcanal :

$$
R_{h}=k \eta L
$$

Une discussion est abordée entre les similitudes d'une telle expression et celle de la résistance d'un barreau métallique de longueur $\mathrm{L}$, de section $\mathrm{S}$ et de résistivité $\rho$ :

$$
R=\frac{\rho L}{S}
$$

La résistivité et la viscosité, responsables de la dissipation d'énergie jouent un rôle analogue. L'ensemble des analogies observées pendant le TD est résumé dans la Table 1.

\begin{tabular}{|c|c|}
\hline Electronique & Microfluidique \\
\hline Potentiel & Pression \\
\hline Intensité & Débit volumique \\
\hline Résistance & Résistance hydraulique \\
\hline Résistivité & Viscosité \\
\hline
\end{tabular}

TABLE 1 - Analogie microfluidique laminaire - électronique

A la fin de la séance, une discussion est menée sur la notion de "nombre de Reynolds". En effet, si la complexité des équations de mécanique des fluides a justifié ici l'utilisation de simulations numériques, d'autres stratégies existent pour caractériser un écoulement sans résoudre analytiquement les équations. L'une d'elles consiste à introduire des nombres sans dimension qualitatifs qui donnent l'importance d'un terme par rapport à un autre dans les équations. En considérant l'équation de Navier-Stokes, le rapport en ordre de grandeur des termes de convection et de diffusion visqueuse donne directement le nombre de Reynolds $R_{e}$ (le calcul est effectué par et avec les étudiants). Ainsi lorsque $R_{e}$ est petit devant 1, l'écoulement est très visqueux, laminaire. En revanche, pour des nombres de Reynolds élevés $\left(R_{e}>100\right)$, la convection est plus importante et des tourbillons peuvent apparaître. Afin de vérifier ces affirmations, la dernière partie du TD consiste à introduire un obstacle dans l'écoulement et à observer celui-ci à différents nombres de Reynolds, en modifiant la pression d'entrée. Les observations sont présentées en Figure 4.

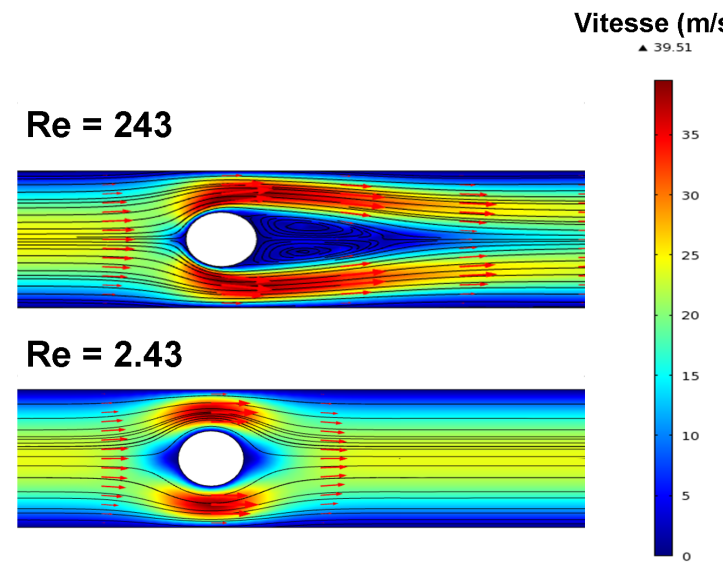

FIgURE 4 - Ecoulements après l'obstacle en fonction du nombre de Reynolds

La présence de recirculations de fluides apparaît pour les plus hauts nombres de Reynolds (qui montrent une limite de l'analogie microfluidique/électronique) alors que l'écoulement à faible nombre de Reynolds est totalement réversible. La troisième séance de TD verra l'introduction d'un autre nombre caractéristique : le nombre de Péclet.

Ainsi cette simulation clôt le TD avec la discussion sur l'écoulement laminaire. Des calculs d'ordre de grandeur du nombre de Reynolds permettent de mettre en évidence la similitude de l'écoulement du miel aux échelles macroscopiques et de l'eau aux échelles microscopiques. Voici la conclusion formulée par un des binômes :

"L'expression selon laquelle l'écoulement de l'eau aux échelles microscopiques est similaire à celui de l'huile ou du miel à notre échelle a sa part de vérité : en effet, pour les écoulements d'eau microscopiques, le nombre de Reynolds est fortement diminué à cause des dimensions de l'écoulement, alors que pour un écoulement de miel, par exemple, à notre échelle, le nombre de Reynolds est lui aussi fortement diminué, mais par sa viscosité."

\section{Capillarité et microfluidique}

Après avoir décrit les différents aspects de l'écoulement laminaire dans le TD1, les étudiants sont amenés à travailler sur les effets de surface et leur influence en microfluidique [8]. L'objectif premier est d'utiliser la simulation comme moyen de compréhension du phénomène physique de montée capillaire. Ensuite, par la simulation, les paramètres en jeu dans ce 
phénomène pourront être évalués.

Après quelques rappels théoriques sur les définitions de l'angle de contact, des caractères hydrophiles ou hydrophobes de surfaces, la loi de Laplace est invoquée pour justifier le phénomène de montée capillaire. En effet, lorsque de l'eau remplit partiellement un canal, le caractère hydrophile des murs du canal entraîne la formation d'un ménisque au niveau de l'interface eau/air, lui même responsable d'une chute de pression par la loi de Laplace (Figure 5). Cette chute de pression entraîne un déplacement du fluide appelé "montée capillaire".

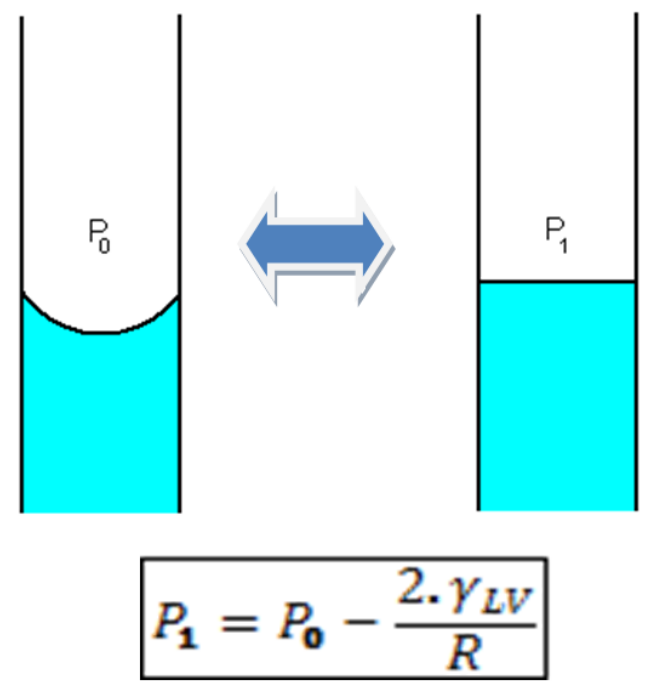

FiguRE 5 - Chute de pression responsable de la montée capillaire. $\gamma_{L V}$ est la tension de surface entre l'eau et l'air et $\mathrm{R}$ le rayon de courbure du ménisque.

Il est ensuite proposé de discuter des caractéristiques de cette montée capillaire (régime transitoire, influence des murs du canal, etc.) grâce à la simulation. L'étude consiste à étudier le comportement de l'eau et de l'air dans un microcanal partiellement rempli. Pour cela, le module "Phase Field" de COMSOL est utilisé et la simulation est effectuée en régime transitoire. L'étude est ici en symétrie de révolution pour simuler le comportement d'un capillaire cylindrique de rayon $5 \mu \mathrm{m}$ et de longueur $200 \mu \mathrm{m}$. Dans un premier temps, la force de gravitation n'est pas prise en compte. Son influence sera étudiée en fin de séance. La simulation de la montée capillaire pendant $1 \mathrm{~ms}$ est représentée en Figure 6.

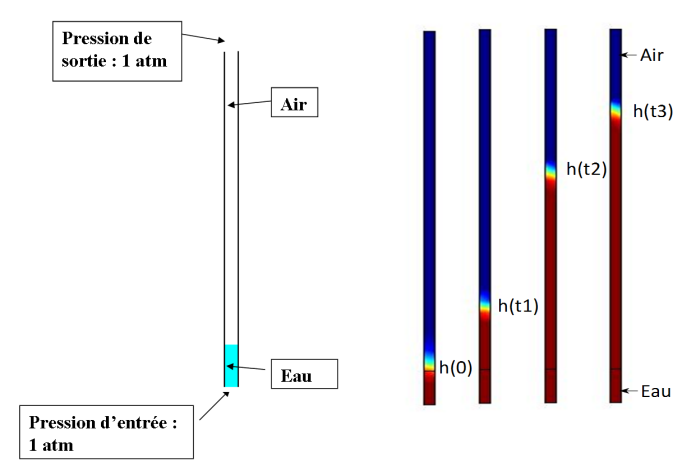

FIgURE 6 - Conditions initiales et simulation de la montée capillaire pendant $1 \mathrm{~ms}$. $h(t)$ représente la position de l'interface.

Afin de mieux comprendre ce phénomène, il est demandé aux étudiants de tracer l'évolution de la pression le long du microcanal. Trois zones sont alors remarquées (Figure 7). Dans la zone 3, la pression est quasiment constante et correspond à l'air. Ensuite un saut de pression est observé au niveau de l'interface entre l'eau et l'air (zone 2), qui correspond à la pression de Laplace. Ce saut de pression est responsable du gradient de pression (zone 1) au sein de l'eau (car l'entrée du canal est à la pression atmosphérique) qui entraîne la montée capillaire, le débit d'eau étant proportionnel au gradient de pression ( $\mathrm{cf}$ TD1).

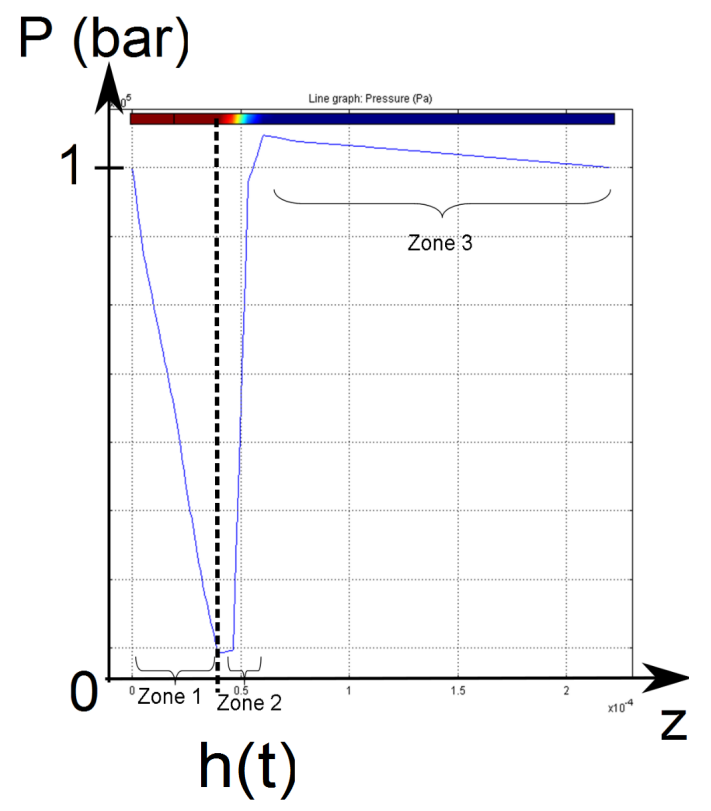

Figure 7 - Profil de pression le long du microcanal 
Or, le liquide avançant et le saut de pression à l'interface étant constant, le gradient de pression diminue et ne devient nul qu'après un temps infini (Figure 8). Ainsi, dans de telles conditions, la montée capillaire ne s'arrête jamais. Le débit décroit sans jamais réellement devenir nul.

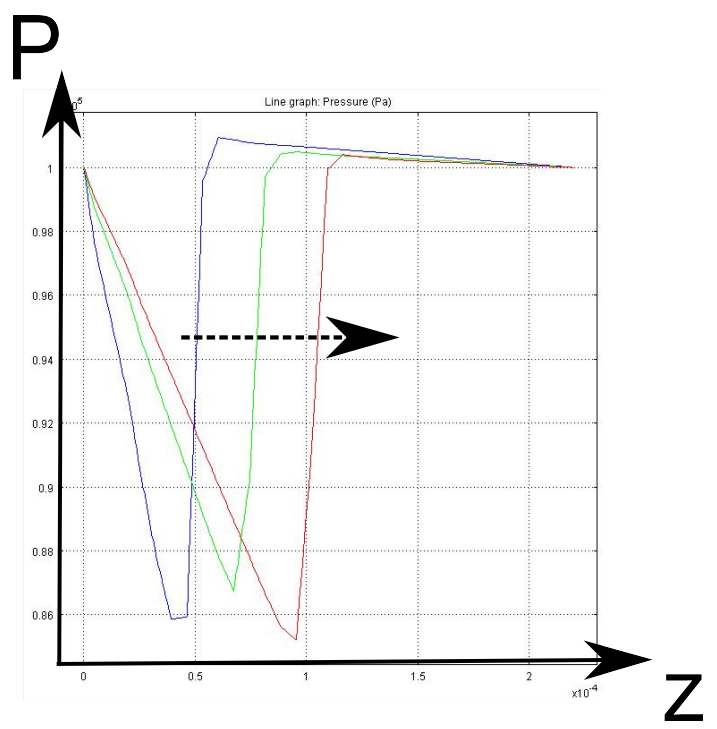

FIgURE 8 - Profil de pression le long du microcanal pour 3 instants différents : avancée du liquide et décroissance du débit.

La simulation précédente permet aux étudiants de bien comprendre le mécanisme physique responsable de la montée capillaire. Une discussion est alors menée avec les étudiants pour justifier le caractère infini de la montée. Dans la réalité, la montée de l'eau crée un gradient de pression hydrostatique qui, lorsqu'il compensera le saut de pression de Laplace, arrêtera la montée capillaire. Cependant, l'effet de la pesanteur continuera d'être négligé dans la suite. A partir des considérations précédentes, les étudiants sont guidés pour modéliser la situation par une équation différentielle. Sa résolution, non traitée pendant le TD, donne la loi de Washburn [9] qui décrit quantitativement l'évolution de la position $h(t)$ de l'interface au cours du temps :

$$
h(t)=\sqrt{\frac{2 \gamma_{L V} a \cos \theta}{4 \eta} t+h^{2}(0)}
$$

où $\theta$ est l'angle de contact du liquide sur le mur, $\eta$ la viscosité du liquide, $a$ le rayon du microcanal et $\gamma_{L V}$ la tension de surface entre l'eau et l'air. Il est alors demandé aux étudiants de déterminer par la simulation l'évolution de la position de l'interface en fonction du temps et en fonction du type de microcanal (hydrophile, hydrophobe). Les résultats sont présentés sur la
Figure 9. Conformément à la loi de Washburn, plus les murs sont hydrophiles, plus la montée est rapide. En revanche, pour les murs hydrophobes, on observe une "descente capillaire".

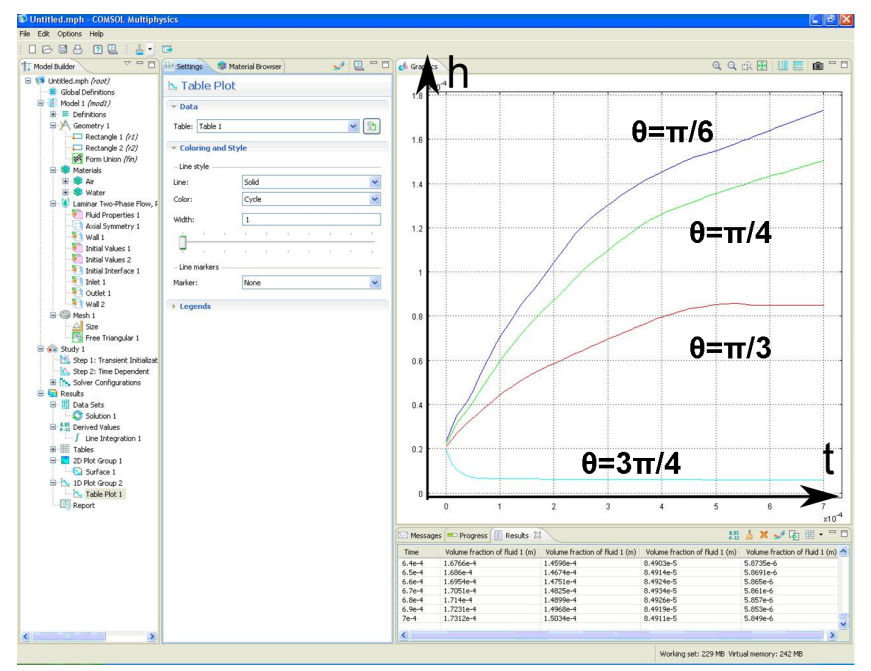

FIgURE 9 - Evolution de l'interface en fonction du temps pour différents angles de contact $\theta$

Dans la dernière partie, l'influence de la pesanteur est simulée et les étudiants observent qu'elle est négligeable. En effet, à l'échelle du microcanal, la surpression " $\rho g h$ " due à la pesanteur est négligeable devant le saut de pression de Laplace.

En guise de conclusion, les applications des effets de surface ont été discutées, comme le déplacement de gouttes par électromouillage $[10,11]$, la mouillabilité des surfaces rugueuses [12] ou les écoulements diphasiques [13]. Voici la conclusion du TD retrouvée dans un compte-rendu : "La montée capillaire est un phénomène dû uniquement aux effets de surface liés à la plus ou moins grande mouillabilité d'un substrat. Elle présente un intérêt énorme, puisqu'elle permet de déplacer un fluide sans apport d'énergie extérieure !"

\section{Mélange dans les microcanaux}

Après avoir abordé les principes du transport par écoulement laminaire dans les microcanaux dans le TD1 et le transport par capillarité dans le TD2, les étudiants sont amenés dans le TD3 à travailler sur une application de la microfluidique pour la chimie. En effet, de nombreux travaux sont menés actuellement afin d'utiliser des microréacteurs pour effectuer des réactions (bio)chimiques [14]. Concrètement, lorsqu'une espèce $A$ réagit avec une espèce $B$ dans un 
microcanal, quelles sont les différences (cinétiques, thermodynamiques) avec la même réaction effectuée dans un bécher?

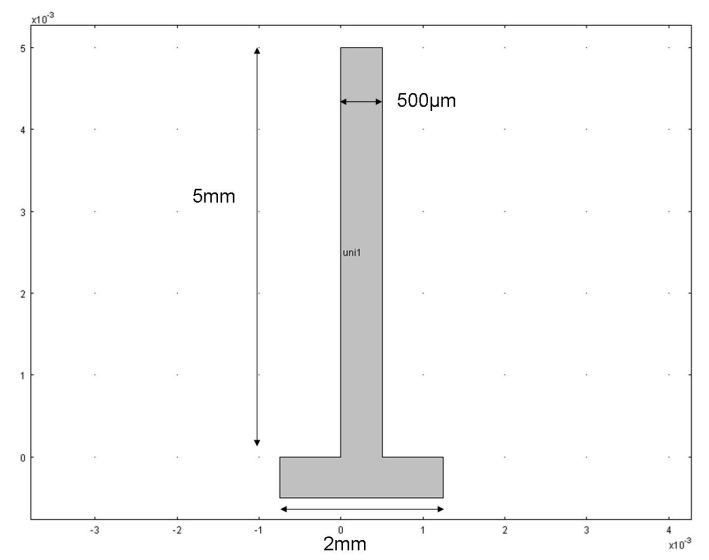

FIGURE 10 - Microréacteur de type jonction T

La structure d'un microréacteur typique est la jonction $\mathrm{T}$ présentée en Figure 10. L'espèce $\mathrm{A}$ entre par l'entrée de gauche et l'espèce $B$ par celle de droite et la réaction a lieu le long du canal central. En réalité, pour qu'il y ait réaction, il faut que les deux expèces se mélangent. A l'échelle macroscopique habituelle, ce mélange est immédiat grâce à la convection : en agitant la solution, l'écoulement devient turbulent et permet aux réactifs de se mélanger (comme le sucre dans le café). En revanche, en microfluidique, on sait que l'écoulement est laminaire, rendant a priori impossible le mélange par convection. C'est ici la diffusion, agitation brownienne des molécules en solution, qui va être responsable du mélange des deux réactifs [15]. Celle-ci nécessite un certain temps et ainsi, les débits dans les microcanaux doivent être choisis suffisamment faibles afin d'assurer le mélange. Le nombre de Péclet est défini comme le rapport entre le temps nécessaire au phénomène de diffusion $\tau_{d i f f}$ et le temps de séjour des réactifs dans le microcanal $\tau_{\text {conv }}$ :

$$
P_{e}=\frac{\tau_{\text {diff }}}{\tau_{\text {conv }}}=L v / D
$$

où $v$ est la vitesse caractéristique du fluide, $L$ la longueur caractéristique de l'écoulement et $D$ le coefficient de diffusion caractéristique des espèces chimiques.

Les objectifs du TD sont de comprendre la prépondérance du phénomène de diffusion en microfluidique et de voir quelles conséquences/applications en terme de mélange et de filtrage elle peut avoir. Les étudiants sont ainsi amenés à simuler la situation pour différents nombres de Péclet. La simulation est effectuée en régime permanent et combine les modules "Laminar Flow" et "Convection and Diffusion". En modifiant les débits d'entrée, la qualité du mélange en fonction du nombre de Péclet est visualisée en Figure 11.

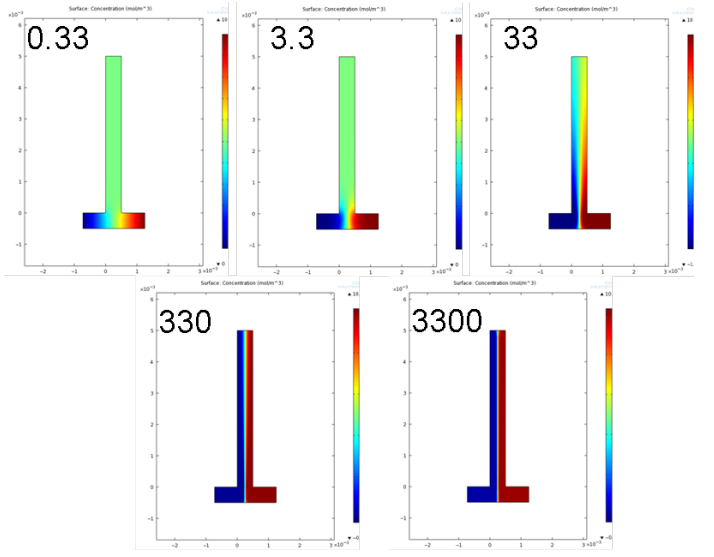

FIgURE 11 - Visualisation du mélange en fonction du nombre de Péclet

La notion de triangle de diffusion est également introduite (Figure 12) pour visualiser la compétition entre diffusion et convection.

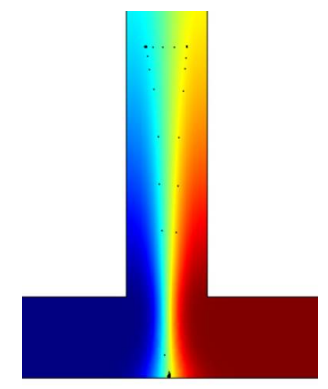

FigURE 12 - Visualisation du triangle de diffusion

Une application d'un tel comportement est le filtrage de particules. En effet, lorsque deux molécules de coefficients de diffusion différents (de taille différente par exemple) sont dans une même solution, la géométrie de type "Filtre en $\mathrm{H}$ " permet d'extraire la molécule de coefficient de diffusion le plus élevé. Un exemple est traité dans le TD avec un mélange alcool/protéine dans lequel on souhaite extraire l'alcool, dont la diffusion est plus forte. Il est représenté en Figure 13.

Les deux molécules sont introduites par l'entrée en bas à droite. L'alcool diffusant suffisamment, il se retrouve en partie dans la sortie 1. En revanche, la protéine étant plus grosse et possédant un coefficient 


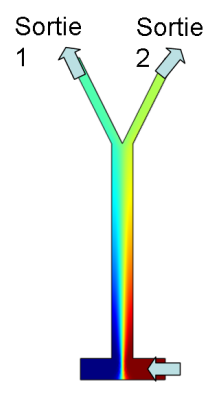

Ethanol

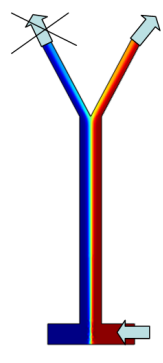

Albumine
FIgURE 13 - Filtre en H pour l'extraction de l'éthanol

de diffusion plus important, elle ne parvient pas à migrer vers la sortie 1 et se retrouve entièrement en sortie 2 . Il est donc possible en récoltant la sortie 1 de filtrer l'éthanol. Une analogie a d'ailleurs été menée avec les étudiants avec le filtrage en électronique. Il est ici possible de tracer un diagramme de type Bode où le paramètre discriminant est le coefficient de diffusion plutôt que la fréquence. Le filtre étudié est alors ici un passe-haut.

Dans l'énoncé du TD, un extrait d'une publication de recherche est proposé où un tel filtre est utilisé pour la séparation de spermatozoïdes motiles et non-motiles en vue d'application en terme de traitement de la stérilité.

Enfin, le TD se conclue sur une perspective pour améliorer l'efficacité des mélanges en microfluidique, basée sur des recirculations de fluides (effet Dean) lorsqu'un microcanal présente une courbure [16]. Les étudiants sont ici amenés à effectuer une simulation 3D dans laquelle ils observent la recirculation du fluide dans une section du microcanal (Figure 14).

De là, il est demandé aux étudiants d'imaginer des structures de mélangeurs qui pourraient être intégrés. Une proposition est présentée en Figure 15, qui correspond d'ailleurs à un choix réalisé en pratique par certaines équipes de recherche.

Voici enfin la conclusion apportée par un groupe à ce TD : "Nous avons vu qu'aux échelles micrométriques, le caractère fortement laminaire des écoulements implique que les mélanges se font nécessairement par diffusion. Le gros intérêt de ce phénomène est qu'il ne nécessite aucun appareillage spécifique (il repose uniquement sur le mouvement brownien) mais le problème réside dans sa durée, qui est très longue et qui implique donc des canaux très longs ou des débits très faibles."

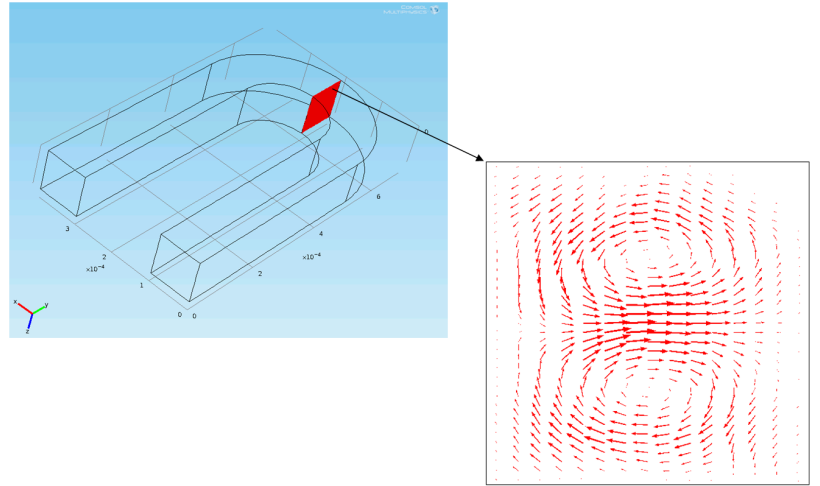

FIgURE 14 - Mise en évidence de la recirculation transverse au sein d'un microcanal courbé

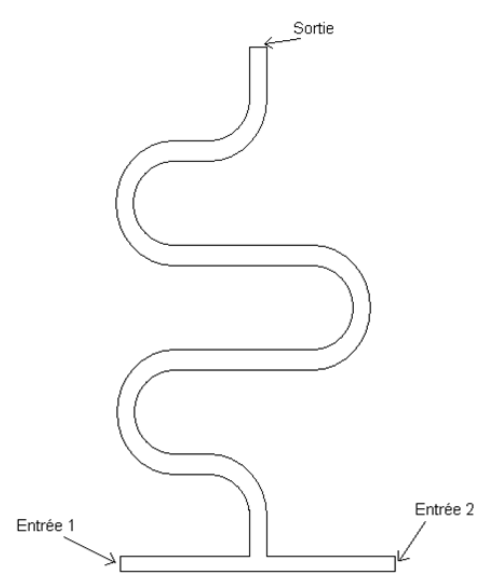

FiguRe 15 - Proposition de mélangeur par les étudiants

Une discussion se poursuit sur un autre phénomène diffusif : le transfert thermique, qui lui aussi possède des propriétés différentes de celles macroscopiques. En effet, dans les microcanaux, il est possible de chauffer ou refroidir de manière rapide un échantillon liquide.

\section{Conclusion}

Ces séances de TD ont permis aux étudiants d'apprendre à utiliser un logiciel de simulation aux éléments finis : COMSOL. La rédaction des énoncés a été réfléchie de manière à laisser les étudiants de plus en plus libres quant à la manière de simuler. En ce qui concerne les connaissances en microfluidique, les thèmes d'écoulement laminaire, de profil parabolique, d'effets capillaires et de diffusion ont été traités et couvrent une grande partie des problématiques de microfluidique. 
Ces TDs ont été accompagnés d'une partie "cours", basée sur la compréhension d'exemples de travaux de recherche et d'applications, ainsi que d'un TP expérimental où les étudiants ont pu réaliser des mélangeurs microfluidiques et visualiser leurs performances.

Bien que d'origines diverses (physique, physique médicale ou EEA), les étudiants semblent avoir bien adhéré aux séances proposées et se sont mobilisés lors des séances de TDs de manière très satisfaisante. Nous espérons pouvoir reconduire plusieurs années cette expérience et, par le biais de cette communication, échanger avec d'autres enseignants concernant ce transfert pédagogique d'une discipline en devenir.

\section{Références}

[1] B. Pascal. Expériences nouvelles touchant le vide. P. Margat, 1647.

[2] B. Pascal. Récit de la grande expérience de l'équilibre des liqueurs. C. Savreux, 1648.

[3] P. Tabeling. Le monde étonnant des mems. XVème congrès français de Mécanique, 2001.

[4] S. Laurette, A. Treizebre, F. Affouard, and B. Bocquet. Subterahertz characterization of ethanol hydration layers by microfluidic system. Applied Physics Letters, 97(11) :111904, 2010.

[5] P. Yager, T. Edwards, E. Fu, K. Helton, K. Nelson, M. R. Tam, and B. H. Weigl. Microfluidic diagnostic technologies for global public health. Nature, 442(7101):412-418, 2006.

[6] Todd M. Squires and Stephen R. Quake. Microfluidics : Fluid physics at the nanoliter scale. Rev. Mod. Phys., 77(3) :977, Oct 2005.

[7] J. Berthier and P. Silberzan. Microfluidics for biotechnology. Artech House, 2010.

[8] R. B. Fair, A. Khlystov, T. D. Tailor, V. Ivanov, R. D. Evans, P. B. Griffin, V. Srinivasan, V. K. Pamula, M. G. Pollack, and J. Zhou. Chemical and biological applications of digital-microfluidic devices. Ieee Design \& Test of Computers, 24(1) :10-24, 2007.

[9] E. W. Washburn. The dynamics of capillary flow. Physical Review, 17(3) :273-283, 1921.

[10] F. Mugele and J.-C. Baret. Electrowetting : from basics to applications. Journal of Physics : Condensed matter, 17 :R705-R774, 2005.
[11] F. Lapierre, G. Piret, H. Drobecq, O. Melnyk, Y. Coffinier, V. Thomy, and R. Boukherroub. High sensitive matrix-free mass spectrometry analysis of peptides using silicon nanowires-based digital microfluidic device. Lab On A Chip, 11(9):16201628, 2011.

[12] R. Dufour, M. Harnois, Y. Coffinier, V. Thomy, R. Boukherroub, and V. Senez. Engineering sticky superomniphobic surfaces on transparent and flexible pdms substrate. Langmuir, 26(22) :17242-17247, 2010.

[13] X. C. I. Solvas and A. deMello. Droplet microfluidics : recent developments and future applications. Chemical Communications, 47(7): 19361942, 2011.

[14] A. Abbas, A. Treizebre, P. Supiot, N. E. Bourzgui, D. Guillochon, D. Vercaigne-Marko, and B. Bocquet. Cold plasma functionalized terahertz biomems for enzyme reaction analysis. Biosensors \& Bioelectronics, 25(1) :154-160, September 2009.

[15] L. E. Locascio. Microfluidic mixing. Analytical and Bioanalytical Chemistry, 379(3) :325-327, 2004.

[16] A. P. Sudarsan and V. M. Ugaz. Fluid mixing in planar spiral microchannels. Lab On A Chip, 6(1) :74-82, 2006. 